\title{
Thermodynamic aspects of an LNG tank in fire and experimental validation
}

\author{
Corina Hulsbosch-Dam*, Bilim Atli-Veltin, Jerry Kamperveen, Han Velthuis, Johan Reinders, Mark Spruijt and Lex \\ Vredeveldt
}

TNO, Van Mourik Broekmanweg 6, 2628 XE Delft, The Netherlands

\begin{abstract}
Mechanical behaviour of a Liquefied Natural Gas (LNG) tank and the thermodynamic behaviour of its containment under extreme heat load - for instance when subjected to external fire source as might occur during an accident - are extremely important when addressing safety concerns. In a scenario where external fire is present and consequent release of LNG from pressure relief valves (PRV) has occurred, escalation of the fire might occur causing difficulty for the fire response teams to approach the tank or to secure the perimeter. If the duration of the tank exposure to fire is known, the PRV opening time can be estimated based on the thermodynamic calculations. In this paper, such an accidental scenario is considered, relevant thermodynamic equations are derived and presented. Moreover, an experiment is performed with liquid nitrogen and the results are compared to the analytical ones. The analytical results match very well with the experimental observations. The resulting analytical models are suitable to be applied to other cryogenic liquids.
\end{abstract}

\section{Introduction}

Liquefied natural gas (LNG) is the cleanest burning fossil fuel and the demand for its use and transportation has been increasing in the last years due to the demanding reduced emission requirements on land and sea. For heavy duty truck fleets use of LNG as fuel is financially and environmentally very attractive, and nowadays many commercial options for vehicles working with LNG are available [1]. Similarly, in marine applications, there is significant number of examples of inland waterway vessels and short sea vehicles mainly in the Netherlands and USA [2]. Meanwhile, risks associated with the storage and transportation of large amounts of cryogenic liquid - not only LNG - have been the topic of discussions. Fire response of LNG tanks is one of the topics that harbour many questions, one of which is the opening time of the pressure relief valves (PRVs). The opening time of the PRV located on an LNG tank that is subjected to external fire is crucial information that fire response teams would like to have before approaching an accident. One of the first steps to reach that information is by investigating the thermodynamics of a cryogenic liquid tank that is subjected to external heat source.

The heat transfer calculation in a scenario where the outside temperatures reach more than $900^{\circ} \mathrm{C}$ and the inner liquid is at $-163^{\circ} \mathrm{C}$ is a complex topic. LNG storage and transport tanks are usually double walled cylindrical structures made of stainless steel. The annular space between the walls is filled with insulation material, in combination with vacuum (low) pressure. The most common tank design consists of a perlite insulation at vacuum pressures. More recent designs, incorporate multilayer insulation (MLI) technology for insulation [3].

The operating pressures of cryogenic tanks are usually below 10 bar. Due to the fact that some heat transfer between the outside and the inside is always present, constant evaporation of the LNG occurs forming boil-off gas (BOG). Therefore, in an LNG tank, almost always vapour and liquid are present. Detailed design of tanks varies with manufacturer; however one of the common target is limiting the number of heat leak points between the inner and outer tanks. Stationary LNG tanks are generally supported with balsa wood located in the insulation space, and mobile tanks have skirts to limit the motion of the inner tank and to transfer the loads of the weight of the inner tank and the LNG to the outer tank.

In case of an external fire, heat transfer to the inner tank, and therefore the BOG, increases. Increasing gas in a limited space causes inner pressures to rise. PRVs are usually set to release this gas for safety reasons, circumventing a violent burst of the tank. In a scenario where external fire is present and consequent release of LNG from PRV has occurred, escalation of the fire might occur causing difficulty for the fire response teams to approach the tank or to secure the perimeter. If the duration of the tank exposure to fire is known, given the intensity of the fire, the PRV opening time can be estimated based on the thermodynamic calculations. In this paper, such a scenario is considered; relevant thermodynamic equations are derived and presented. Moreover, an experiment is performed with liquid

Corresponding author: corina.hulsbosch@tno.nl

C The Authors, published by EDP Sciences. This is an open access article distributed under the terms of the Creative Commons Attribution License 4.0 (http://creativecommons.org/licenses/by/4.0/). 
nitrogen and the results are compared to the analytical ones. The resulting analytical models are suitable to be applied to other cryogenic liquids.

\section{Experiments}

Any tank filled with refrigerated or liquefied gas is susceptible to heat influx, due to the storage conditions and physical properties of the substance inside the tank. Natural gas (methane) has to be refrigerated below its critical point of $-82.6^{\circ} \mathrm{C}$ (at 45 bar) to be liquefied to LNG (approx. $-163^{\circ} \mathrm{C}$ at 1 bar). Transport and storage tanks for cryogenic fluids (e.g. LNG) are provided with thermal insulation to prevent or at least minimize the heat influx from a source in the surroundings - ambient air, solar radiation, and fire exposure - into the liquid. A temperature increase of the liquid will result in a pressure increase in the tank. Pressures above the maximum allowed working pressure (MAWP) should be prevented by activation of installed PRV. In general, a PRV is a spring loaded safety valve. Furthermore, the yield and tensile strengths of the pressurized shell, carbon or stainless steel construction, will start to decrease at temperatures above $300^{\circ} \mathrm{C}$ [4]. Progressive weakening of the shell strength could lead to catastrophic tank rupture above its design and test pressures.

The resistance of a pressurized tank against fire exposure cannot be demonstrated by separately testing the elements of the fire protection system. The tank construction (wall thickness, insulation) and its safety accessories (heat resistant coating, double walls, PRV) need to be tested as a whole and subjected to heat radiation or direct flame impingement in order to bring the stress conditions on materials. Heat load tests where the tank is fully engulfed in flames are called bonfire tests. In 2015, a bonfire test has been performed by TNO at BAM facilities in Germany [5]. The goal of this test was to study the thermodynamic and mechanical behaviour of a partially filled tank in fire.

A double-walled tank with an inner volume of $3 \mathrm{~m}^{3}$ (inner diameter: $1.2 \mathrm{~m}$, outer diameter: $1.6 \mathrm{~m}$; length: $2.384 \mathrm{~m}$ ) was placed in fire. The tank consisted of an inner tank and an outer tank. The test tank was made of low temperature resistant stainless steel (type 304) to contain cryogenic fluids (LIN, LNG, etc.). The wall thickness for both tanks was $3 \mathrm{~mm}$, which is thinner than for commercially available tanks $(\sim 6 \mathrm{~mm})$. The thickness of the end caps of the outer tank were $5 \mathrm{~mm}$. This also reduces the maximum allowed working pressure from 10 bar to 7 bar. A drawing of the tank can be seen in Fig. 1.

The inner and outer tanks were separated by $20 \mathrm{~cm}$ of perlite, which was the insulation material chosen for this experiment. The volume filled with perlite was vacuumed to 200 mbar. (For commercial tanks this annular space is usually $\sim 10$ mbar or below, in which case the thermal conductivity improves significantly.) The pressure inside the tank was monitored during the test. In addition, the temperature of the outer tank wall was monitored. Furthermore, thermocouples are placed to measure the temperature of the fluid (3 locations) and the inner wall (7 locations).

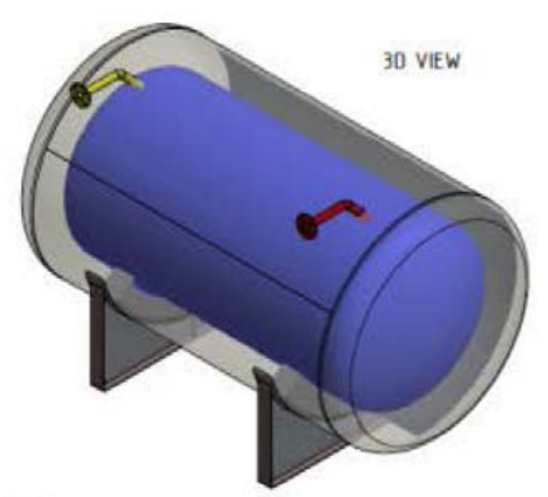

Fig. 1. 3D schematic view of the double wall tank used at the fire experiments

For safety, liquid nitrogen (LIN) was used in the experiments described in the current paper. The tank was filled with $1400 \mathrm{~kg}$ of LIN, which corresponds to a filling level of $66 \%$. The filling level of the tank was controlled by the instrumentation on the filling truck as well as the load cells placed under the tank. After some settling time, the fire was initiated and the tank was completely engulfed in fire within seconds. The flame temperatures reached up to $800-900{ }^{\circ} \mathrm{C}$ at the bottom of the tank, and $450-600{ }^{\circ} \mathrm{C}$ at the top. An impression of the test is given in Fig. 2.

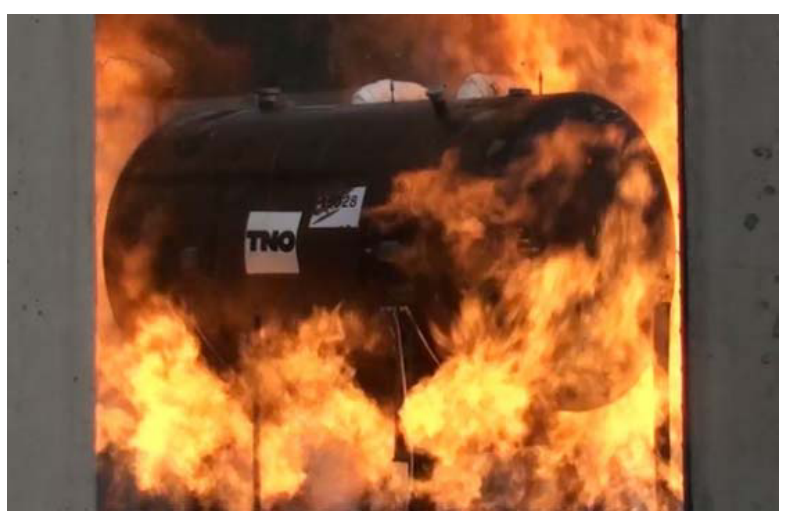

Fig. 2. Double walled tank containing cryogenic liquid engulfed in fire during the experiment

A double walled LNG tank that is engulfed in fire will have an outer tank temperature that is beyond $600^{\circ} \mathrm{C}$ within minutes from the start of the fire. Mechanical strength of the mild and stainless steel at these high temperatures is poor. Depending on the compressive performance of the insulation layer and the presence (or not) of the stiffening rings on the outer tanks interior, buckling of the outer tank is the most likely failure mode of the outer tank. The driving force to such an event is the vacuum (very low pressure) on the interior face and 
the atmospheric pressure on the exterior face of the external tank. The subsequent behaviour of the tank depends on the scenario; for instance a case where the PRV is not working properly, or there is damage on the inner tank which may result in different behaviour. In case of an accidental failure of the outer tank and a consequent loss of vacuum (or any other cause to an increase of the tank's overall thermal conductivity) the LNG in the inner tank will warm up faster than during normal use, and therefore its vapour pressure will increase faster. Considering such a scenario, the failure mode of the inner tank could be burst or rupture, at a weak spot due to increase of the inner pressure. Before performing the experiments, the burst pressure of the tank was calculated, and the tests were stopped when the inner pressures reached a predetermined value, which was below the calculated burst pressure.

\section{Numerical models}

Two different numerical models are described. The first is an equilibrium model which assumes that the vapour and liquid in the tank have the same temperature and pressure. The second model is a non-equilibrium model which allows temperature difference between the liquid and the vapour. The non-equilibrium model is the more realistic model of the two, however, it also requires more input parameters.

The developed models, in principle, can be applied to any (cryogenic) substance such as LIN or LNG. In order to be able to compare the results of the models to the experiments, the properties of LIN were used in the numerical models. The models use thermodynamic properties of LIN (and LNG) which were taken from the NIST database [6] for the equilibrium model and from the coolprop database [7] for the non-equilibrium model. The following section describes the difference of these two conditions.

\subsection{Equilibrium model}

The simplest model to describe the tank is an equilibrium model in which the liquid and vapor are considered as one node, i.e. the liquid and vapor have the same temperature and pressure. A schematic representation is given in Fig. 3. In this figure, $m$ is the mass inside the tank, $V$ is the tank volume, $T$ is the temperature of the tank contents, $p$ is the pressure inside the tank, $m_{\text {out }}$ and $h_{\text {out }}$ are the mass and the enthalpy leave the system through the PRV, respectively, and $Q_{\text {in }}$ is the heat input into the tank.

For this system both mass and energy conservation are applied (neglecting changes in tank volume and assuming no mass leaves the tank):

$$
\begin{gathered}
\mathrm{d} m / \mathrm{d} t=0 \\
\mathrm{~d}(m u) / \mathrm{d} t=Q_{i n},
\end{gathered}
$$

in which $t$ is time and $u$ is the internal energy. These equations are only valid until the PRV opens and mass starts to leave the system.

The heat input depends on the fire temperature and the insulation properties. As a first step, a simple approximation can be taken as:

$$
Q_{\text {in }}=(A k / d)\left(T_{\text {fire }}-T_{\text {tank }}\right)
$$

in which $A$ is the surface area of the tank, $k$ is the thermal conductivity, $d$ is the thickness of the insulation material, $T_{\text {fire }}$ is the flame temperature and $T_{\text {tank }}$ is the temperature inside the tank.

Having no mass leaving the tank and no volume changes of the tank results in a overall density in the tank which is constant in time. The heating process of the tank can then be described by following the corresponding constant density line in an pressureenthalpy diagram.

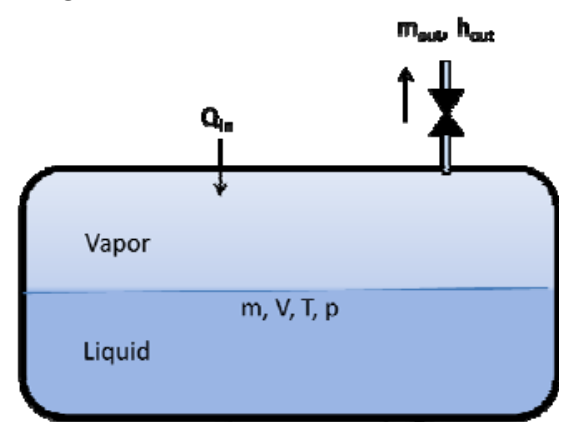

Fig. 3. Equilibrium tank model

The numerical relations for the equilibrium case that are described above were modelled at TNO in Matlab commercial code. This model takes less than 20 seconds to run. The main steps of this TNO model are summarized in the schematic below:

Input of the variables:

Wall thickness, insulation value, tank dimensions, fire temperature, PRV opening pressure, filling level

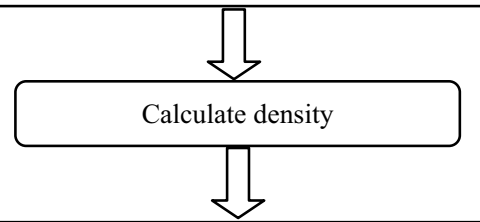

Fetch the corresponding pressure vs enthalpy data of from the NIST database for the specific density

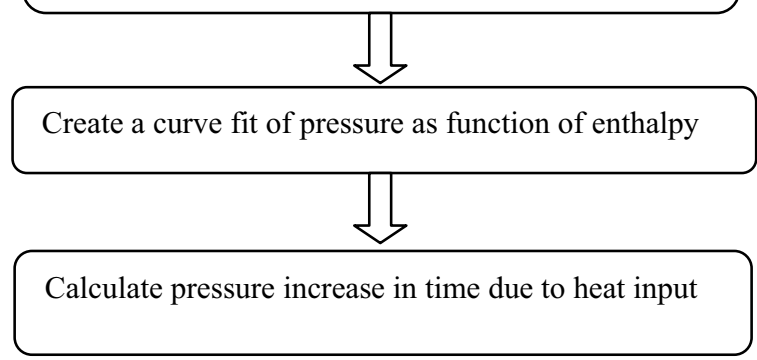




\subsection{Non-equilibrium model}

The non-equilibrium model consists of several nodes, similar to the one shown in Fig. 4. The vapour and liquid are described as separate nodes, also the heat transfer through the tank inner and outer wall is taken into account.

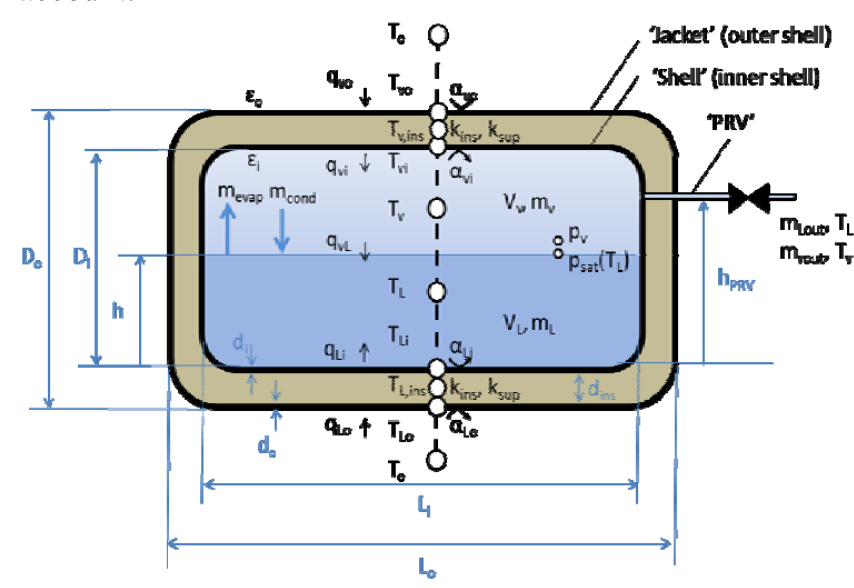

Fig. 4. Non-equilibrium tank model including tank walls and insulation.

For the vapour, liquid and total fluid mass the conservation of mass and energy are considered, including mass transfer between the vapour and liquid phase. This results in 4 equations for 5 unknowns: liquid and vapour mass: $m_{L}, m_{v}$; liquid and vapor temperature: $T_{L}, T_{v}$; and the pressure: $p_{v}$. To complete the set of equations the conservation of total volume is added:

$$
V_{L}+V_{v}=\text { constant }
$$

The set of equations has to be supplemented with expressions for the liquid and vapor discharge rates, the evaporation/condensation rates and the energy sources due to wall or interface interactions. For the evaporation/condensation rate $\left(\dot{m}_{n e t}\right)$ the HertzKnudsen-Langmuir-equation is used:

$$
\dot{m}_{\text {net }}=A \cdot \sqrt{\frac{M_{v}}{2 \pi \cdot R}} \cdot\left(\beta_{e} \cdot \frac{p_{s a t}\left(T_{L}\right)}{\sqrt{T_{L}}}-\beta_{c} \cdot \frac{p_{v}}{\sqrt{T_{v}}}\right)
$$

in which the evaporation and condensation coefficients, $\beta_{e}$ and $\beta_{e}$, can be used to better fit the experimental data with the numerical model. The default value of 1 for both coefficients is used.

The energy sources in the conservation equations due to wall or interface reactions can further be described by several other processes. The first is the heat input of the fire, which can be described as radiation, or temperature or a combination of radiation and temperature. The walls of the tank will heat up and will release heat to the adjacent fluid.

As long as the fluid is quiescent, the heat is transferred, to the fluid and inside the fluid, through conduction. When the density differences in the liquid increase, a gravity driven flow pattern develops. This leads to a free convective heat transfer near the tank walls which depends on the Nusselt number.
The non-equilibrium model is programmed in Mathcad and takes 30 seconds to 1 minute to run.

\section{Results and Discussions}

During the first 118 minutes of the exposure to the fire the pressure inside the tank increased from 2.0 bar to 7.6 bar, at which pressure the pressure relieve valve (PRV) opened. The fuel supply for the fire was switched off 122 minutes after it started. An image of the tank after the fire has been switched off is shown in Figure 5. Some buckling of the outer wall can be seen.

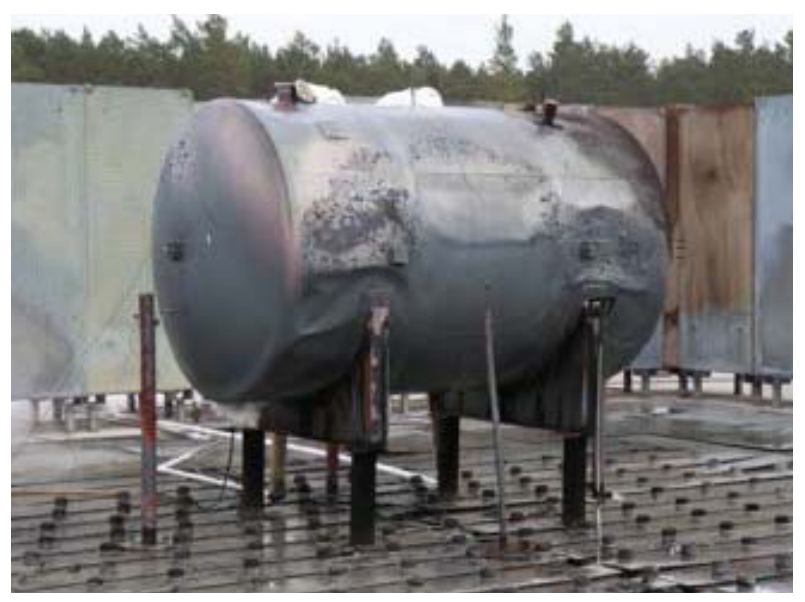

Fig. 5. Cryogenic tank after the fire test

The pressure increase over time during the fire experiment is shown in Fig. 6 with the solid line. The dashed and the dotted line represent the pressure curves obtained by the non-equilibrium model and the equilibrium model respectively. In this plot, $t=0 \mathrm{~min}$ corresponds to the start of the fire. At this point the pressure starts to slowly increase and after 40 minutes it starts to rise faster and after $120 \mathrm{~min}$ the pressure reaches 7.6 bar. At this point PRV opens and subsequently the pressure remains constant until the fire is switched off and the pressure decreases again.

The initial flat portion of the curve indicates a stable period in the heating during which it is safe for firefighters to approach the tank and extinguish the external fire. The rapid increase in pressure in the later stage is where it becomes more dangerous to approach the LNG tank in during an external fire due to the risk of mechanical failure (and explosion) or the opening of the PRV. 


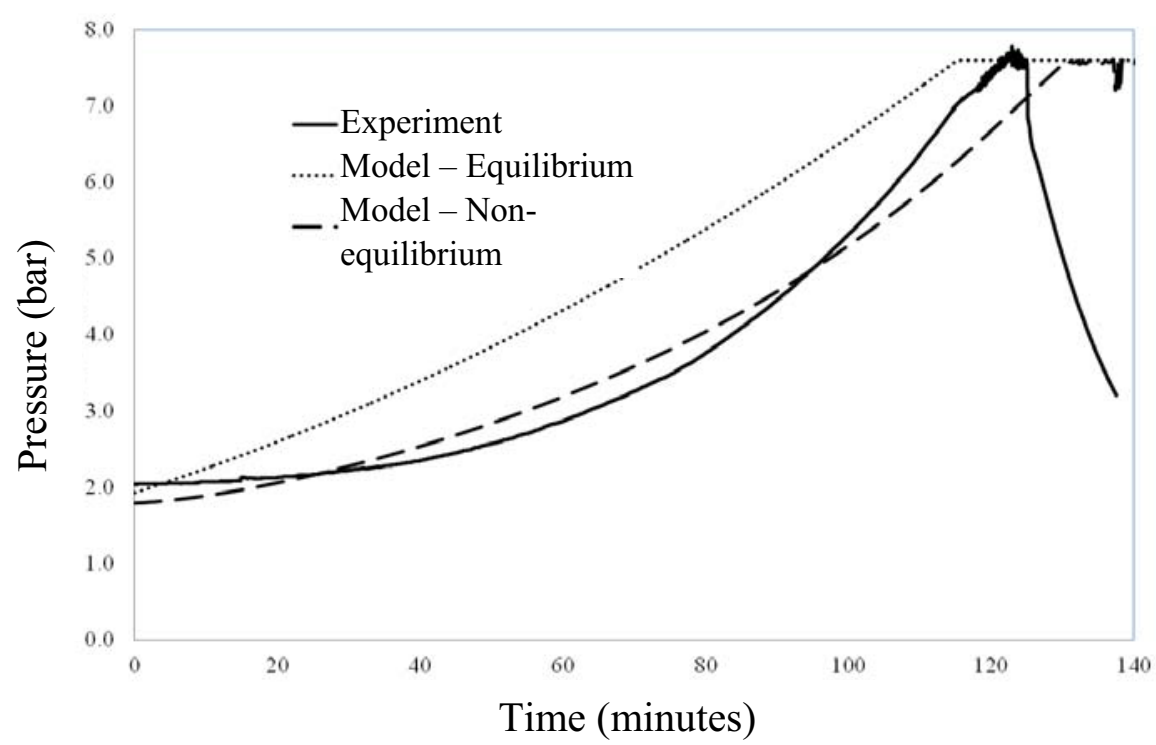

Fig. 6. Pressure vs time curves for the experiments, non-equilibrium model and equilibrium model.

Both the equilibrium and non-equilibrium models are able to capture the PRV opening time with acceptable error. The non-equilibrium model gives a better description of the slope of the pressure increase. The slow increase in pressure and temperature of the fluid directly at the start of the fire is caused by the heating of the wall and insulation before an effective heat transfer to the fluid starts. This delays the increase in temperature of the fluid, as is shown by the small slope at the start of the test. In the equilibrium model, the heating of the walls and the insulation are not included. For this reason the increase of temperature and pressure is nearly constant over time and doesn't show a speed up. The main parameter for this model is the heat input into the tank $\left(Q_{i n}\right)$. For the shown curve the heat input into the tank is taken constant from the beginning to the end. The model can be improved by using the time dependent tank temperature and temperature dependent heat conductivity. Adding these improvements allows a better match to the final slope of the experiments, however the initial slow heat up can't be properly captured by this model. The non-equilibrium model is the most complete model, and however, it also has most number of equations and needs several coefficients to describe the process. The actual solution is dependent on many coefficients and parameters. Some of which are: $\beta_{e}$ and $\beta_{e}$ for the evaporation/condensation model and thermal conductivity, $k$.

\section{Conclusions}

Fire response of cryogenic tanks was studied experimentally and numerically in this investigation. Experimental approach is based on subjecting a double walled, perlite insulated tank with 1.4 tons of liquid nitrogen to external fire until the pressure valve openings were observed. The numerical modelling are based on developing two models; one for assumption of equilibrium between the liquid and the vapour which was developed in Matlab and the second one assumed non-equilibrium and was developed in Mathcad.

The pressure evolution during the fire experiment was recorded and the compared to the numerical results. The increase in pressure starts slowly and at a later point starts to increase. The initial phase is used to heat up the tank itself and the insulation between the inner and outer wall. Both of the numerical models, capture the "Time to release" with small error. Two models have been described: equilibrium and non-equilibrium. The equilibrium model is able to describe the final, fast heat up of the fluid. However, as the thermal mass of the walls and insulation is not included in the model, the initial slow heat up is not captured well. The nonequilibrium model is able to describe both phases in the heat up and is able to follow the experimental results very well. In order to better validate the developed model more experimental data are needed. This can be data on LNG or LIN or any other cryogenic substance.

This work and the models developed by TNO can be used by the fire response teams to estimate the time to approach an accident area involving a cryogenic tank such as LNG fuel or transport tanks.

\section{Acknowledgements}

The Dutch Ministry of Economic Affairs (Topsector Energy, TKI gas program) and Joint Industry Partners namely Rolande LNG, Engie, Shell, and Vopak are kindly acknowledged for their financial support.

TNO performed the experiments described in this paper at the test facility of BAM, the Bundesanstalt für Materialforschung und Prüfung (Berlin/Horstwalde, Germany). Special thanks go to their dedicated on site operational team. 


\section{References}

1. U.S. Department of Energy. [Online] https://www.portofrotterdam.com/en/cargoindustry/lng-liquefied-natural-gas/lng-as-a-fuel-forvessels-and-trucks.

2. Port of Rotterdam. [Online] https://www.portofrotterdam.com/en/cargoindustry/lng-liquefied-natural-gas/lng-as-a-fuel-forvessels-and-trucks.

3. R.G. Scurlock, Stratification, Rollover and Handling of LNG, LPG and Other Cryogenic Liquid Mixtures. London: Springer, (2016). ISSN 21915520.

4. J.F.M. Velthuis, and B. Atli-Veltin, Thermal and Mechanical Model of a Vacuum Insulated Cryogenic Tank Subjected to External Tank. Delft: TNO R10940, (2015).

5. J.P. Kamperveen, M.P.N. Spruijt, and J.E.A Reinders,. Heat Load Resistance of Cryogenic Storage Tanks - Results of LNG Safety Program. Utrecht : TNO, (2016).

6. NIST. National Institute of Standards and Technology. [Online] http://www.nist.gov/.

7. I.H. Bell, J. Wronski, S. Quoilin, V. Lemort,. 53, (2014), I\&ECR, pp. 2498-2508. 